\title{
Uso de corticoides por cirurgiões bucomaxilofaciais do Brasil
}

\section{Use of corticoids by oral and maxillofacial surgeons in Brazil}

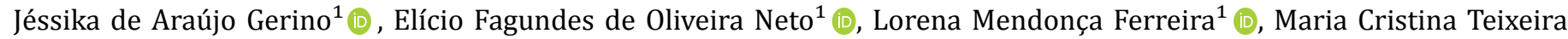 \\ Cangussu $^{2}$ (10) , Sandra de Cássia Santana Sardinha ${ }^{2,3}$ (10, Weber Céo Cavalcante ${ }^{2,3}$ (D)
}

1. Discente do curso de Odontologia pela Universidade Federal da Bahia (UFBA), Salvador, BA, Brasil. 2. Docente do curso de Odontologia pela Universidade Federal da Bahia (UFBA), Salvador, BA, Brasil. 3. Preceptor da Residência em Cirurgia e Traumatologia Buco-Maxilo-Facial do Hospital Santo Antônio - Obras Sociais Irmã Dulce (OSID), Salvador, BA, Brasil.

\section{Resumo}

Objetivo: verificar a frequência, os tipos e os momentos em que os corticoides são utilizados nos procedimentos como cirurgias de dentes inclusos, cirurgias de instalação de implantes, cirurgias reconstrutivas para implantes, cirurgias ortognáticas, cirurgias de trauma de face e cirurgias de patologias realizadas por especialistas em Cirurgia e Traumatologia Bucomaxilo-Facial (CTBMF) do Brasil. Métodos: 0 estudo foi realizado mediante uma pesquisa quantitativa em que se utilizou um questionário composto por 25 perguntas aplicado a 70 especialistas em Cirurgia e Traumatologia Bucomaxilofacial que compareceram a um evento científico do Colégio Brasileiro de Cirurgia e Traumatologia BucoMaxiloFacial. Resultados: a utilização de corticoides foi frequente em todos os procedimentos investigados no questionário, principalmente nas cirurgias ortognáticas em que $100 \%$ dos participantes confirmaram o uso. A variação nos regimes de uso de corticoides foi significativa. Constatou-se o uso de diferentes tipos de corticoides, sendo a dexametasona o fármaco que prevaleceu. Quanto às doses, percebeu-se, também, grande variação entre as respostas. Conclusão: em geral, os corticoides, da forma como são, frequentemente, administrados pelos cirurgiões bucomaxilofaciais nos diversos procedimentos cirúrgicos, parecem ser seguros, sobretudo no pré-operatório de cirurgias ortognáticas; porém, sua efetividade pós-operatória ainda é questionável.

Palavras-chave: Corticoides. Cirurgia Bucal. Procedimentos Cirúrgicos Operatórios.

\begin{abstract}
Objective: to verify the frequency, types and moments that corticosteroids are used in procedures such as included teeth surgeries, implant installation surgeries, reconstructive implant surgeries, orthognathic surgeries, facial trauma surgeries and surgeries of pathologies performed by specialists in Oral Maxillofacial Surgery and Traumatology - Facial (CTBMF) from Brazil. Methods: The study was conducted through a quantitative survey using a questionnaire consisting of twenty-five questions applied to seventy specialists in Oral Maxillofacial Surgery and Traumatology - Facial who attended a scientific event of the Brazilian College of Oral Maxillofacial Surgery and Traumatology - Facial. Results: The use of corticosteroids was frequent in all procedures investigated in the questionnaire, mainly in orthognathic surgeries in which $100 \%$ of the participants confirmed the use. The variation in corticosteroids use regimes was significant. The use of different types of corticosteroids was detected, with dexamethasone being the drug that prevailed. As for the doses, there was also a great variation among the responses. Conclusion: In general, corticosteroids as they are often administered by oral maxillofacial surgeons in various surgical procedures seem to be safe especially in the preoperative period of orthognathic surgery, but their postoperative effectiveness is still questionable.
\end{abstract}

Keywords: Corticosteroids. Oral Surgery. Operative Surgical Procedures.

\section{INTRODUÇÃO}

Os corticoides são hormônios produzidos pelas glândulas suprarrenais que possuem forte ação anti-inflamatória e são amplamente utilizados em procedimentos cirúrgicos na região maxilofacial ${ }^{1,2}$.

A administração de corticoides no pré, trans ou pós-operatório de cirurgias maxilofaciais são usualmente realizadas na tentativa de reduzir dor, edema, trismo, náuseas, vômitos e promover recuperação neurossenssorial ${ }^{3,4}$. Os corticoides mais utilizados são a dexametasona, metilprednisolona e betametasona, aplicados por via oral, intramuscular e endovenosa ${ }^{3}$.

A utilização de corticoides de forma inadequada pode comprometer a saúde do paciente, tendo em vista os efeitos adversos aos quais podem levar, como supressão da glândula suprarrenal, aumento do índice de infecção, catarata, glaucoma, hipertensão, miopatia, osteoporose, alterações no humor ou personalidade e psicose ${ }^{3-5}$. Os corticoides administrados por via oral ou endovenosa são mais propensos a causar esses efeitos adversos do que os de uso tópico ou inalatório ${ }^{1}$.

Os reais benefícios da utilização de corticoides em procedimentos cirúrgicos realizados pelos cirurgiões bucomaxilofaciais deixam espaço para dúvidas quanto às dosagens, ao momento de utilização, à via de administração, e, até mesmo, a própria efetividade da droga. Desse modo, talvez, a utilização desse popular medicamento possa não estar sendo a mais adequada ${ }^{2-6}$.

Este estudo se propõe a verificar a frequência, os tipos 
e os momentos em que os corticoides são utilizados nos procedimentos, como cirurgias de dentes inclusos, cirurgias de instalação de implantes, cirurgias reconstrutivas para implantes, cirurgias ortognáticas, cirurgias de trauma de face e cirurgias de patologias realizadas por especialistas em cirurgia e traumatologia bucomaxilofacial do Brasil, e, se há algum padrão em sua utilização no intuito de provocar discussão que possa estimular a reflexão e aproximar-se do uso racional desses fármacos.

\section{MÉTODOS}

O presente estudo foi aprovado pelo Comitê de Ética em Pesquisa em Seres Humanos da Faculdade de Odontologia da Universidade Federal da Bahia sob número: 88288218.9.0000.5024. Todos os participantes foram devidamente orientados a ler e a assinar o Termo de Consentimento Livre e Esclarecido (TCLE), caso aceitassem participar da pesquisa.

Foi realizado um estudo transversal, utilizando-se um questionário como instrumento para sua realização, contendo 25 perguntas relacionadas ao uso de corticoides. As perguntas consideradas como base deste estudo foram: "Você utiliza corticoides perioperatórios nos seus procedimentos cirúrgicos? De que modo e em quais tipos de cirurgia?"

O questionário não foi validado, porém o estudo prévio utilizado foi o artigo: "A survey of the use of perioperative glucocorticoids in oral and maxillofacial surgery" publicado pela revista Journal of Oral and Maxillofacial Surgery no ano de 2016, com o código doi:10.1016/j.joms.2016.02.027, que pesquisou o assunto que o presente estudo havia pesquisado entre cirurgiões bucomaxilofaciais de outro país.

A coleta de dados foi realizada pelo pesquisador responsável e por dois acadêmicos do curso de Odontologia, os quais foram devidamente calibrados para o processo da entrevista e para possíveis discussões acerca das questões relacionadas ao sigilo.

Foram incluídos, no estudo, 70 especialistas em Cirurgia e Traumatologia Buco Maxilofacial (CTBMF) presentes em um evento científico do Colégio Brasileiro de Cirurgia e Traumatologia BucoMaxiloFacial que aceitaram responder, presencialmente, ao questionário instrumento deste estudo. Aqueles também informaram o local onde concluíram sua especialização e o tempo de conclusão. Foram excluídos os não especialistas em Cirurgia e Traumatologia Bucomaxilofacial.

O entrevistado foi informado pelo pesquisador de que não existia intenção de verificar respostas certas ou erradas, no intuito de encorajar o entrevistado a fornecer respostas mais coerentes à pesquisa proposta.

As 25 perguntas do questionário foram relacionadas aos procedimentos cirúrgicos realizados, incluindo a frequência, os tipos e os momentos em que os corticoides são utilizados em cada procedimento.

Os procedimentos investigados no questionário foram os seguintes: 1) cirurgias de dentes inclusos, 2) cirurgias de instalação de implantes, 3) cirurgias reconstrutivas para implantes, 4) cirurgias ortognáticas, 5) cirurgias de trauma facial e 6) cirurgias de patologias. Para a frequência de utilização dos corticoides, as opções foram: 1) sempre, 2) nunca, 3) na maioria das vezes, 4) na minoria das vezes (em casos selecionados).

As opções de cada fármaco, utilizadas nos procedimentos, foram: 1) hidrocortisona, 2) dexametasona, 3) metilprednisolona, 4) betametasona, 5) prednisolona. E as opções de momento foram: 1) pré-operatório, 2) pós-operatório, 3) transoperatório.

Os participantes também foram convidados a relatar, no questionário, quaisquer complicações relacionadas ao uso de corticoides em sua experiência clínica.

O processamento dos dados foi realizado em uma planilha eletrônica no programa Excel, e a análise estatística, feita em pacote estatístico MINITAB, versão 14. Foram realizadas análises descritivas de frequência absoluta e relativa, medidas de tendência central e variabilidade das variáveis de interesse. Em seguida, foram desenvolvidas as análises estratificadas para uma avaliação preliminar de potenciais associações, que foram estimadas associações brutas (Razões de Prevalência e Intervalo de Confiança a 95\% obtidos pelo Método Wald) entre as variáveis. Para as variáveis contínuas, foi utilizado o teste $\mathrm{t}$ de Student e, para as variáveis categoriais, o qui-quadrado; ambos com nível de significância de $5 \%$.

\section{RESULTADOS}

Dos 70 entrevistados, 67,2\% concluíram a especialização em até dez anos. A faixa etária predominante foi de 31 a 40 anos $(59,4 \%)$, e a prevalência foi do sexo masculino $(92,8 \%)$, sendo que nem todos os entrevistados responderam a todas as perguntas do questionário.

Mesmo o evento científico sendo realizado na região Nordeste do país, $29 \%$ dos entrevistados eram de regiões distintas, como Sudeste e Sul. A diferença regional entre os entrevistados não exerceu influência sobre a prática na administração de corticoides. O surgimento de alguma complicação, ao longo da experiência profissional, foi relatado por nove entrevistados (13\%), variando entre soluço, pele oleosa em mulheres, surgimento de acne, urticária, alergia e insuficiência renal. (tabela 1)

A maioria dos cirurgiões entrevistados utiliza corticoides com grande frequência. Foi constatado que, dos 70 entrevistados, 55 (78,6\%) alegaram usar corticoides em cirurgia de dentes inclusos; 41 (58,6\%) fazem uso de corticoides em cirurgia de instalação de implantes; 53 (75,7\%) em cirurgia reconstrutiva de implantes; 70 (100\%) em cirurgia ortognática; 58 (82,9\%) em cirurgia de trauma; 49 (70\%) em cirurgia de patologia. (tabela 2) 
Tabela 1. Informações gerais dos entrevistados. Salvador/BA, 2019

\begin{tabular}{lrr}
\hline Variáveis & N & \% \\
\hline Estado de origem & 25 & 37,0 \\
Bahia & 14 & 21,0 \\
Pernambuco & 5 & 7,0 \\
Ceará & 2 & 3,0 \\
Paraíba & 2 & 3,0 \\
Piauí & 14 & 21,0 \\
São Paulo & 5 & 7,0 \\
Rio de Janeiro & 1 & 1,0 \\
Rio Grande do Sul & &
\end{tabular}

\section{Sexo}

$\begin{array}{lrr}\text { Masculino } & 64 & 92,8 \\ \text { Feminino } & 5 & 7,2\end{array}$

$\begin{array}{lrl}\text { Idade } & & \\ \text { De } 27 \text { a } 30 \text { anos } & 12 & 17,4 \\ \text { De } 31 \text { a } 40 \text { anos } & 41 & 59,4 \\ \text { De } 41 \text { a } 50 \text { anos } & 11 & 15,9 \\ \text { Mais de } 50 \text { anos } & 5 & 7,27\end{array}$

Tempo de especialização

\begin{tabular}{lrr} 
Até 10 anos & 45 & 67,2 \\
De 11 a 20 anos & 16 & 23,8 \\
De 21 a 30 anos & 5 & 7,8 \\
Mais de 30 anos & 1 & 1,5 \\
Complicações & & \\
Não & 61 & 87 \\
Sim & 9 & 13 \\
\hline
\end{tabular}

Entre os tipos de corticoides listados no questionário, a dexametasona foi o fármaco de escolha prevalente entre os 70 entrevistados, com doses de 4, 6, 8 e 10mg, relacionadas com cada tipo de procedimento e com o momento de utilização, seguido de hidrocortisona e prednisolona.

A utilização de corticoides nos diferentes tempos cirúrgicos dos procedimentos analisados no questionário foi em cirurgia de dentes inclusos: 60 entrevistados utilizam corticoides no pré-operatório e, desses, 25 entrevistados também utilizam no pós-operatório e um utiliza, também, no transoperatório. Cinco entrevistados só utilizam corticoides no pós-operatório, e, desses, um utiliza, também, no transoperatório, e somente um entrevistado utiliza corticoide apenas no transoperatório.

Em cirurgia de instalação de implantes: 58 entrevistados utilizam corticoides no pré-operatório. Desses, 20 entrevistados também utilizam no pós-operatório, e dois utilizam, também, no transoperatório; três entrevistados só utilizam corticoides no pós-operatório, e nenhum entrevistado utiliza corticoide apenas no transoperatório.

Em cirurgia reconstrutiva para implantes: 59 entrevistados utilizam corticoides no pré-operatório. Desses, 25 entrevistados também utilizam no pós-operatório e dois utilizam também no transoperatório. Cinco entrevistados só utilizam corticoides no pós-operatório, e nenhum entrevistado utiliza corticoides apenas no transoperatório.

Em cirurgia ortognática: 48 entrevistados utilizam corticoides no pré-operatório. Desses, 39 entrevistados também utilizam no pós-operatório, e 31 utilizam, também, no transoperatório. Dezesseteentrevistadossóutilizam corticoidesnopós-operatório e cinco entrevistados utilizam apenas no transoperatório. Um viés pode ser observado quanto à utilização dos corticoides no pré e transoperatório de cirurgias maiores, como a ortognática, a cirurgia de trauma e de patologias.

Em cirurgia de trauma: 47 entrevistados utilizam corticoides no pré-operatório. Desses, 29 entrevistados também utilizam no pós-operatório e 20 utilizam, também, no transoperatório. Quatorze entrevistados só utilizam corticoides no pósoperatório e seis utilizam apenas no transoperatório.

Em cirurgia de patologias: 39 entrevistados utilizam corticoides no pré-operatório. Desses, 23 entrevistados, também, utilizam no pós-operatório e 14 utilizam, também, no transoperatório. Quatorze entrevistados só utilizam corticoides no pós-operatório e sete entrevistados utilizam apenas no transoperatório. (tabela 2)

Tabela 2. Uso dos corticoides nos diferentes procedimentos e tempos cirúrgicos. Salvador/BA, 2019.

\begin{tabular}{lrrrrrrrrrr}
\hline \multirow{2}{*}{ Tipo de cirurgia } & \multicolumn{3}{c}{ Não/ minoria } & \multicolumn{1}{c}{ Usa/ maioria } & \multicolumn{2}{c}{ Pré } & \multicolumn{3}{c}{ Pós } & \multicolumn{3}{c}{ Trans } \\
\cline { 2 - 12 } & $\mathbf{n}$ & $\%$ & $\mathbf{n}$ & $\%$ & $\mathbf{n}$ & $\%$ & $\mathbf{n}$ & $\%$ & $\mathbf{n}$ & $\%$ \\
\hline Dentes inclusos & 15 & 21,4 & 55 & 78,6 & 60 & 90,9 & 30 & 45,4 & 4 & 6,0 \\
Instalação de implantes & 29 & 41,4 & 41 & 58,6 & 58 & 95,1 & 23 & 37,7 & 2 & 3,3 \\
Reconstrutiva de implantes & 17 & 24,3 & 53 & 75,7 & 59 & 92,2 & 30 & 46,9 & 2 & 3,1 \\
Cirurgia ortognática & 2 & 2,9 & 68 & 97,1 & 48 & 68,6 & 56 & 80 & 36 & 51,4 \\
Trauma & 12 & 17,1 & 58 & 82,9 & 47 & 70,1 & 43 & 63,2 & 26 & 38,2 \\
Patologia & 21 & 30 & 49 & 70 & 39 & 65 & 37 & 61,6 & 21 & 35 \\
\hline
\end{tabular}




\section{DISCUSSÃO}

Os resultados apontaram que a maioria dos entrevistados administram corticoides nos procedimentos cirúrgicos listados. Em relação ao uso de corticoides em cirurgia de dentes inclusos, foi encontrada, no presente estudo, uma maior uniformidade entre o padrão de utilização por parte dos entrevistados e a literatura; o resultado da pesquisa apontou que $90,9 \%$ dos entrevistados usam corticoides no pré-operatório, concordando com estudos como Larsen et al., $(2018)^{5}$ que, por meio de uma revisão sistemática de literatura, avaliaram diferentes dosagens de corticoides em cirurgia de terceiro molar impactado e concluíram que a administração pré-operatória de corticoide diminui, significativamente, o edema facial após a remoção cirúrgica de terceiro molar impactado, mas não houve uma dosagem ideal, e a revisão sistemática não focou no tempo de administração.

Markiewicz et. al $(2018)^{6}$, por intermédio de uma meta-análise, avaliaram o efeito de corticoides no controle do edema, da dor e do trismo pós-operatório precoce e tardio, após remoção de terceiro molar. Os autores concluíram que a administração perioperatória de corticoides produz uma redução do edema, da dor e melhora na abertura bucal, após a remoção cirúrgica de terceiros molares, concordando com a escolha de $78,6 \%$ dos cirurgiões bucomaxilofaciais entrevistados neste trabalho, que fazem uso de corticoides em cirurgias de dentes inclusos, sendo que $90,9 \%$ usam no pré-operatório, $45,4 \%$ no pós-operatório e apenas $6,0 \%$ no transoperatório.

Bahammam et al. $(2016)^{7}$, em um ensaio clínico, randomizado, duplo-cego e controlado, analisaram a administração de corticoides no pré-operatório e no pós-operatório de cirurgia de instalação de implantes e concluíram que o uso da dexametasona é eficaz para prevenir ou controlar a dor e o desconforto pós-operatórios, após a instalação do implante.

Nesta pesquisa, foi observado que mais da metade dos entrevistados $(58,6 \%)$ utilizam corticoides em cirurgias de instalação de implantes, sendo que 95,1\% utilizam no préoperatório e $37,7 \%$ utilizam no pós-operatório, estando essa conduta sustentada pelo trabalho de Bahammam et al., (2016) ${ }^{7}$.

Em relação às cirurgias reconstrutivas de implantes, não foram encontrados estudos na literatura que confirmassem a efetividade dos corticoides nesse tipo de cirurgia. No presente estudo, mesmo que $75,7 \%$ dos entrevistados afirmem utilizar corticoides em cirurgias reconstrutivas de implantes, eles não justificaram o porquê do seu uso.

No presente estudo, $100 \%$ dos entrevistados relataram utilizar corticoides em cirurgias ortognáticas, visto que $68,6 \%$ usam no pré-operatório e $80 \%$ usam no pós-operatório. Porém, o uso no pós-operatório não se adapta ao estudo de Chegini e Dhariwal $(2012)^{8}$, que realizaram uma revisão de literatura sobre uso de corticoides em cirurgia ortognática, cujo objetivo é estabelecer a relação entre o tipo e a dose de corticoides necessários para a redução do edema.

De acordo com o estudo, existe benefício no uso pré-operatório; porém, o uso pós-operatório é controverso, havendo pouca evidência sobre uma dose terapêutica ideal. Entende-se que os dados coletados neste trabalho, referentes ao uso préoperatório em cirurgia ortognática, podem não representar os valores reais, pois a alegação de diversos cirurgiões bucomaxilofacias entrevistados foi de que a administração de corticoides pré-operatórios é realizada pelo anestesista, não podendo precisar a utilização ou não de corticoides no préoperatório.

Snall et al. $(2015)^{9}$ realizaram estudo randomizado para esclarecer os benefícios da dexametasona no período perioperatório na consolidação tardia de fratura de mandíbula. Concluiu-se que a dexametasona, em altas doses, em curto prazo, predispõe à consolidação complicada da fratura, especialmente em pacientes com fraturas angulares de mandíbula. Já Kormi et al. (2017) ${ }^{10}$ avaliaram a eficácia analgésica da dexametasona em sua utilização perioperatória, indicando eficiência no objetivo de reduzir a percepção de dor associada ao uso de dexametasona; porém, em seu estudo, não é avaliada nos diferentes tempos cirúrgicos. Dessa forma, a escolha dos profissionais entrevistados no presente estudo está bem embasada quanto à terapêutica, visto que dos 58 participantes, (82,9\%) utilizam corticoides em cirurgias de trauma. Porém, a utilização por parte dos profissionais ainda não está bem esclarecida, visto que 70,1\% entre eles utilizam no pré-operatório, pois o uso no pós-operatório $(63,2 \%)$ e no transoperatório $(38,2 \%)$ não encontrou respaldo literário que comprovasse seus benefícios.

Kormi et al $(2016)^{2}$ afirmam, mediante uma pesquisa por questionário, que a maioria dos profissionais $(85,2 \%)$ administram corticoides perioperatórios em altas doses por curto período, nos procedimentos cirúrgicos oral e maxilofacial. O uso de corticoides nos diferentes procedimentos cirúrgicos difere muito, sendo usados por todos os profissionais em $100 \%$ dos casos para a cirurgia ortognática, do mesmo modo que no presente estudo. Em cirurgias de trauma facial, 43,5\% dos profissionais administram corticoide no estudo de Kormi et al. $(2016)^{2}$, sendo uma porcentagem menor do que o encontrado neste trabalho, pois observa-se administração de corticoides por $82,9 \%$ dos entrevistados. Foi observado que, apesar do respaldo na literatura para o uso de corticoides em cirurgias de terceiro molar, indicando dor e edema reduzidos no pósoperatório, os profissionais participantes do estudo raramente indicavam corticoides para esse tipo de utilização, indo contra o presente estudo em que $78,6 \%$ dos entrevistados administram corticoides em cirurgias de dentes inclusos. Em comparação com o estudo de Kormi et al (2016) ${ }^{2}$, constatou-se, neste estudo, maior utilização de corticoide pelos cirurgiões bucomaxilofaciais em diferentes procedimentos cirúrgicos, exceto em cirurgia ortognática em que os valores encontrados coincidem com Kormi et al. (2016) ${ }^{2}$. Ainda se observou, em Kormi et al. (2016) uma divergência entre a utilização de corticoide e a literatura 
científica, divergência também observada no presente estudo.

Os regimes do uso de corticoides perioperatórios administrados pelos participantes deste estudo tiveram ampla variação. Isso remete ainda a uma lacuna nas pesquisas sobre o assunto, ou seja, faltam estudos que revelem protocolos confiáveis para a utilização de corticoides no que diz respeito à dosagem e ao momento de utilização nos diferentes procedimentos cirúrgicos.

São necessários, provavelmente, ensaios clínicos bem delineados referentes ao uso de corticoides em cirurgia oral e maxilofacial; em especial, os benefícios do uso de corticoides em cirurgias de patologia e em cirurgias de trauma que apresentam poucos estudos.

\section{CONCLUSÃO}

A utilização dos corticoides nos pré-operatórios por parte dos cirurgiões bucomaxilo-faciais está sendo embasada pela literatura na maioria dos procedimentos cirúrgicos, sobretudo em cirurgias ortognáticas; porém, sua efetividade pós-operatória ainda é questionável. Em geral, os corticoides da forma como são frequentemente utilizados por cirurgiões bucomaxilofaciais parecem ser seguros.

\section{REFERÊNCIAS}

1. Mundell L, Lindemann R, Douglas J. Monitoring long-term oral corticosteroids. BMJ open Qual. 2017; 6(2): e000209. doi:10.1136/bmjoq-2017-000209.

2. Kormi E, Snäll J, Törnwall J, Thorén H. A Survey of the Use of Perioperative Glucocorticoids in Oral and Maxillofacial Surgery. J Oral Maxillofac Surg. 2016; 74(8): 1548-1551. doi:10.1016/j.joms.2016.02.027.

3. Dan AEB, Thygesen TH, Pinholt EM. Corticosteroid administration in oral and orthognathic surgery: A systematic review of the literature and meta-analysis. J Oral Maxillofac Surg. 2010; 68(9): 2207-2220. doi:10.1016/j.joms.2010.04.019.

4. Gersema L, Baker K. Use of corticosteroids in oral surgery. J Oral Maxillofac Surg. 1992; 50(3): 270-277. doi:10.1016/0278-2391(92)90325-T.

5. Larsen MK, Kofod T, Christiansen A-E, Starch-Jensen T. Different Dosages of Corticosteroid and Routes of Administration in Mandibular Third Molar Surgery: a Systematic Review. J Oral Maxillofac Res. 2018; 9(2): 1-20. doi:10.5037/ jomr.2018.9201.

6. Markiewicz MR, Brady MF, Ding EL, Dodson TB. Corticosteroids Reduce Postoperative Morbidity After Third Molar Surgery: A Systematic Review and
Meta-Analysis. J Oral Maxillofac Surg. 2008; 66(9):1881-1894. doi:10.1016/j. joms.2008.04.022.

7. Bahammam MA, Kayal RA, Alasmari DS, Attia MS, Bahammam LA, Hassan $\mathrm{MH}$, et al. Comparison Between Dexamethasone and Ibuprofen for Postoperative Pain Prevention and Control After Surgical Implant Placement: A Double-Masked, Parallel-Group, Placebo-Controlled Randomized Clinical Trial. J Periodontol. 2017; 88(1): 69-77. doi:10.1902/jop.2016.160353.

8. Chegini S, Dhariwal DK. Review of evidence for the use of steroids in orthognathic surgery. $\mathrm{Br} J$ Oral Maxillofac Surg. 2012; 50(2): 97-101. doi:10.1016/j.bjoms.2010.11.019.

9. Snäll J, Apajalahti S, Suominen AL, Törnwall J, Thorén A. Influence of perioperative dexamethasone on delayed union in mandibular fractures: A clinical and radiological study. Med Oral Patol Oral Cir Bucal. 2015; 20(5): e621-e626. doi:10.4317/medoral.20553.

10. Kormi E, Snäll J, Koivusalo AM, Suominen AL, Thorén H, Törnwall J. Analgesic Effect of Perioperative Systemic Dexamethasone on Blowout Fracture Surgery. J Oral Maxillofac Surg. 2017; 75(6):1232-1237. doi:10.1016/j.joms.2016.09.026.

Como citar este artigo/How to cite this article:

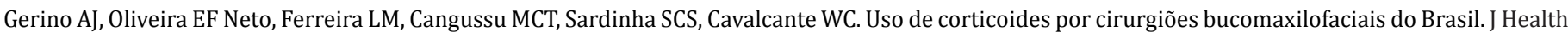
Biol Sci. 2020 J; 8(1):1-5. 